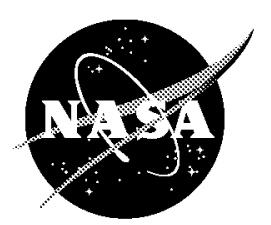

Verification Assessment of Flow Boundary Conditions for CFD Analysis of Supersonic Inlet Flows

John W. Slater

Glenn Research Center, Cleveland, Ohio 
The NASA STI Program Office ... in Profile

Since its founding, NASA has been dedicated to the advancement of aeronautics and space science. The NASA Scientific and Technical Information (STI) Program Office plays a key part in helping NASA maintain this important role.

The NASA STI Program Office is operated by Langley Research Center, the Lead Center for NASA's scientific and technical information. The NASA STI Program Office provides access to the NASA STI Database, the largest collection of aeronautical and space science STI in the world. The Program Office is also NASA's institutional mechanism for disseminating the results of its research and development activities. These results are published by NASA in the NASA STI Report Series, which includes the following report types:

- TECHNICAL PUBLICATION. Reports of completed research or a major significant phase of research that present the results of NASA programs and include extensive data or theoretical analysis. Includes compilations of significant scientific and technical data and information deemed to be of continuing reference value. NASA's counterpart of peerreviewed formal professional papers but has less stringent limitations on manuscript length and extent of graphic presentations.

- TECHNICAL MEMORANDUM. Scientific and technical findings that are preliminary or of specialized interest, e.g., quick release reports, working papers, and bibliographies that contain minimal annotation. Does not contain extensive analysis.

- CONTRACTOR REPORT. Scientific and technical findings by NASA-sponsored contractors and grantees.
- CONFERENCE PUBLICATION. Collected papers from scientific and technical conferences, symposia, seminars, or other meetings sponsored or cosponsored by NASA.

- SPECIAL PUBLICATION. Scientific, technical, or historical information from NASA programs, projects, and missions, often concerned with subjects having substantial public interest.

- TECHNICAL TRANSLATION. Englishlanguage translations of foreign scientific and technical material pertinent to NASA's mission.

Specialized services that complement the STI Program Office's diverse offerings include creating custom thesauri, building customized data bases, organizing and publishing research results... even providing videos.

For more information about the NASA STI Program Office, see the following:

- Access the NASA STI Program Home Page at http://wwo.sti.nasa.gov

- E-mail your question via the Internet to help@stinasa.gov

- Fax your question to the NASA Access Help Desk at 301-621-0134

- Telephone the NASA Access Help Desk at 301-621-0390

- Write to: NASA Access Help Desk NASA Center for AeroSpace Information 7121 Standard Drive Hanover, MD 21076 


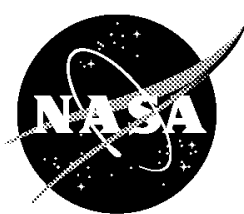

\section{Verification Assessment of Flow Boundary Conditions for CFD Analysis of Supersonic Inlet Flows}

John W. Slater

Glenn Research Center, Cleveland, Ohio

Prepared for the

37 th Joint Propulsion Conference and Exhibit

cosponsored by the AIAA, SAE, AIChE, and ASME

Salt Lake City, Utah, July 8-11, 2001

National Aeronautics and

Space Administration

Glenn Research Center 
The Aerospace Propulsion and Power Program at NASA Glenn Research Center sponsored this work.

Available from

NASA Center for Aerospace Information 7121 Standard Drive

Hanover, MD 21076
National Technical Information Service 5285 Port Royal Road Springfield, VA 22100

Available electronically at http://gltrs.grcnasa.gov 


\title{
VERIFICATION ASSESSMENT OF FLOW BOUNDARY CONDITIONS FOR CFD ANALYSIS OF SUPERSONIC INLET FLOWS
}

\author{
John W. Slater* \\ National Aeronautics and Space Administration \\ Glenn Research Center \\ Cleveland, Ohio 44135
}

\begin{abstract}
$\underline{\text { Abstract }}$
Boundary conditions for subsonic inflow, bleed, and subsonic outflow as implemented into the WIND CFD code are assessed with respect to verification for steady and unsteady flows associated with supersonic inlets. Verification procedures include grid convergence studies and comparisons to analytical data. The objective is to examine errors, limitations, capabilities, and behavior of the boundary conditions. Computational studies were performed on configurations derived from a "parameterized" supersonic inlet. These include steady supersonic flows with normal and oblique shocks, steady subsonic flow in a diffuser, and unsteady flow with the propagation and reflection of an acoustic disturbance.
\end{abstract}

\section{$\underline{\text { Introduction }}$}

The use of gas turbine engines for aircraft capable of supersonic flight requires inlets that decelerate and compress the supersonic flow to subsonic conditions for intake into the engine. The flow delivered to the engine must be of high quality and directed in the axis of the engine. The flow is characterized by supersonic flow with strong shock waves interacting with turbulent boundary layers in an adverse pressure gradient with transition to subsonic flow. The performance of such an inlet is measured in terms of maximizing the total pressure recovery and minimizing the total pressure distortion at the entrance to the engine. The use of flow control devices such as porous bleed holes or slots is one approach for achieving the desired performance by removing low-energy flow in the boundary layer that is susceptible to separation and stabilizing shock motion.

Such inlet flows are being analyzed at the NASA Glenn Research Center (GRC) using computational fluid dynamics (CFD). A flow domain or control volume is defined about the inlet for which the flow field is computed. Boundary conditions must be specified at the boundary of the flow domain. The CFD analysis or simulation is highly dependent on the boundary conditions because the flow is internal and the boundary conditions are applied in proximity to the complex flow features.

The boundary of the flow domain coincides with the surfaces of the inlet and the specified extent of the external and internal flow. The extent of the flow domain is often limited and approximations are introduced to keep the size and complexity of the flow domain at the minimum needed to obtain an accurate solution with the available computational resources.

The flow domain usually includes some of the flow exterior to the inlet intake. The external flow boundaries can often be located close to the intake of the inlet through consideration of the wave nature of supersonic flow. The boundary conditions for these external boundaries are typically fixed inflow or extrapolated outflow, which are applied in a straightforward manner. These boundary conditions are not discussed in this paper.

The flow domain for some simulations may consist only of the subsonic diffuser, which usually starts at the throat of the inlet and extends to the compressor face. This requires a subsonic inflow boundary condition for the inflow boundary. Such simulations may examine the effects of boundary layer blockage on the flow in the subsonic diffuser. Thus the subsonic inflow boundary conditions must have the capability to impose an inflow boundary layer. Such a boundary condition is discussed in this paper.

The flow domain typically does not include the small and complex geometric details of bleed slots or holes. Rather, a boundary condition is applied to model the effects of the bleed. The bleed slots or holes are typically located on the internal surfaces of the inlet with the core flow tangent to the boundary. Three bleed models are discussed and applied in this paper.

*Aerospace Engineer, Inlet Branch. AIAA Senior Member. 
The flow domain typically terminates near the compressor face to avoid the complexity of modeling the geometry and dynamics of the compressor. This requires the application of a subsonic outflow boundary condition. This paper discusses five models for the subsonic outflow boundary condition.

The first objective of this paper is to describe the subsonic inflow, bleed, and subsonic outflow boundary conditions that have been implemented into the WIND CFD code and applied for the analysis of flows through supersonic inlets. ${ }^{1,2}$

The second objective of the paper is to present results of computational studies addressing the verification of these boundary conditions. The studies involve geometric configurations that are derived from a "parameterized" supersonic inlet for which the geometry and grid can be easily generated. This allows a large number of simulations to be performed for a variety of geometries and flow conditions, which strengthens the verification process.

The next sections discuss the verification assessment procedures, the WIND CFD code, the boundary conditions, and the "parameterized" verification inlet. The computational studies are then discussed and include uniform supersonic flow with and without a normal shock, an oblique shock / bleed interaction, subsonic diffuser duct, and an annular duct with unsteady flow of acoustic disturbances.

\section{CFD Verification Assessment Procedures}

Verification in the field of CFD is defined as

\begin{abstract}
The process of determining that a model implementation accurately represents the developer's conceptual description of the model and the solution to the model. ${ }^{3}$
\end{abstract}

This is in contrast to validation, which examines whether the models simulate real-world physics. Verification has its basis in comparison to analytical data, whereas validation has its basis in comparison to experimental data.

The procedures of the verification assessment follow the guidelines of the $\mathrm{AIAA}^{3}$ and the methods as discussed by Roache. ${ }^{4}$ Verification examines 1) if the computational models are the correct implementation of the conceptual models, and 2) if the resulting code can be properly used for an analysis. The strategy is to identify and quantify the errors in the code and the solution. Thus, the two aspects of verification are the verification of a code and the verification of a solution. The verification of a code involves error evaluation, that is, looking for errors in the coding (i.e., bugs) and incorrect implementations of conceptual models. The verification of a solution involves error estimation, that is, determining the accuracy of a calculation and putting an error band on the observed quantity. This paper is focused on the verification of the subsonic inflow, bleed, and subsonic outflow boundary conditions under conditions common to those in supersonic inlets.

The verification assessment assumes that the simulation has reached iterative convergence. For a steady-state flow simulation, the reduction and stabilization of the level of the residual of the flow equations was one measure of iterative convergence. Another measure was the approach of the values of the duct mass flow, bleed mass flow, total pressure recovery to asymptotic values with increasing number of iterations. The iterations were continued for a simulation until the bleed mass flow percentage was invariant to the third decimal place or the massweighted total pressure recovery was invariant to the fourth decimal place.

Verification assessment involves performing consistency checks. One such check is that mass is conserved through the flow domain. For inlets and ducts mass conservation can be assessed spatially along the streamwise coordinate of the duct. Mass flow bookkeeping tracks the mass flow through bleed regions and the compressor face with that of the captured mass flow.

Verification has its basis in comparison to analytical data. Analytical data for verification of the boundary conditions can be obtained from the constraints of the model. The mass flow bleed model should preserve the specified mass flow. Under the uniform conditions, the porous bleed models should provide the mass flow as indicated by the empirical relation for the specified bleed geometry and flow conditions. The boundary conditions are indirectly verified through comparison of the simulation results to available analytic results for the flow field. For the studies discussed here, there are several analytic results for steady, inviscid flow. For an inviscid, subsonic flow, the total temperature and total pressure should be held through the duct. For flow with shocks and turbulent flow the total pressure should decrease through the duct.

One approach for verification of a solution is the grid convergence study, which is a method for determining the "ordered" discretization error in a CFD solution. It involves performing the simulation on two or more successively finer grids. The method results in an error band on the computational result, which indicates the possible difference between the discrete and continuum value. The study assumes that the grid is sufficiently refined such that the solution is in the asymptotic range of convergence, which is the range in which the discretization error reduces asymptotically with decreasing grid size. The objective is to determine the level at which the solution is invariant to the grid. 
The grids of this work were generated using a characteristic grid spacing $h$. The finest grid spacing is denoted as $h_{1}$. For each grid, the simulation results in an observed flow quantity $f$, such as pressure recovery. The change in the quantity $f$ between the grids is expressed in terms of the grid convergence index $(G C I){ }^{4}$ The $G C I$ between a finer grid with spacing $h_{l}$ and coarser grid with spacing $h_{2}$ is defined as

$$
G C I=\frac{F_{s}\left|\left(f_{2}-f_{1}\right) / f_{1}\right|}{r^{p}-1}
$$

where $r$ is the refinement ratio between the finer grid and coarser grid

$$
r=h_{2} / h_{1}
$$

and $p$ is the order of grid convergence observed in the simulations

$$
p=\ln \left(\frac{f_{3}-f_{2}}{f_{2}-f_{1}}\right) / \ln (r) .
$$

The $F_{s}$ is the factor-of-safety. A value of $F_{s}=1.25$ was used in this work. A second-order solution would have $p=2$. The $G C I$ is a measure of the percentage difference of the computed quantity from the value of the asymptotic numerical value; it approximates an error band. It also indicates how much the solution would change with further refinement of the grid. Further details on conducting a grid convergence study can be found in the book by Roache and on the NPARC Verification and Validation web site. ${ }^{\mathbf{4 , 5}}$

\section{WIND CFD Code}

The WIND CFD code is being developed by the NPARC Alliance (National Program for Applicationsoriented Research in CFD), which is a partnership of the NASA Glenn Research Center, the Air Force's Arnold Engineering Development Center, and the Boeing Company. ${ }^{1,2}$ WIND solves the time-dependent, Reynolds-averaged Navier-Stokes equations for turbulent, compressible flows using a cell-vertex, finitevolume, time-marching approach on multi-zone, structured grids. Spatial accuracy is nominally secondorder using the Roe flux-difference splitting upwind formulation. Steady flows are simulated through an iterative process using local time stepping. Unsteady flows are simulated through a second-order marching in time. Turbulence is modeled using one- or twoequation eddy viscosity models. The Spalart-Allmaras and SST models are the most-often-used models.
WIND is capable of solving for flows of speeds ranging from low subsonic to hypersonic. The following three sections describe the boundary conditions that are assessed in this paper.

\section{Subsonic Inflow Boundary Condition}

A subsonic inflow boundary requires four physical conditions to be specified and one numerical condition to be evaluated from the interior of the flow domain. The choice of how the four physical conditions are specified and the numerical condition evaluated characterize the boundary condition. Here our choice is to specify the local total pressure $p_{t}$, total temperature $T_{t}$, and flow angles $\alpha$ and $\beta$ as the physical conditions. Such conditions are consistent with an inflow from a plenum, boundary layer profile, or freestream.

A Newton iteration of the form

$$
T^{m+1}=T^{m}-f / f^{\prime}
$$

is used to converge on the value of static temperature $T$ at the boundary. The function $f$ is derived from the definition of the total temperature

$$
f=T_{t}-T-\frac{\gamma-1}{2} \frac{V^{2}}{\gamma R}
$$

and approaches zero as the iteration converges. The differentiation of $f$ with respect to $T$ is

$$
f^{\prime}=-(1+V / A c)
$$

The functions $f$ and $f^{\prime}$ and the speed of sound $c$ are all only functions of static temperature $T$. The $V$ is magnitude of the velocity $\vec{v}$. The $A$ comes from the expression

$$
\vec{v} \cdot \widehat{n}=V\left(a_{1} \hat{i}+a_{2} \hat{j}+a_{3} \hat{k}\right) \cdot\left(n_{1} \hat{i}+n_{2} \hat{j}+n_{3} \hat{k}\right)=V A
$$

where $a_{1}, a_{2}$, and $a_{3}$ are the direction cosines of the velocity and are evaluated by the expressions

$$
\begin{gathered}
a_{1}=\left(1+\tan ^{2} \alpha+\tan ^{2} \beta\right)^{-1 / 2} \\
a_{2}=\tan \alpha\left(1+\tan ^{2} \alpha+\tan ^{2} \beta\right)^{-1 / 2} \\
a_{3}=\tan \beta\left(1+\tan ^{2} \alpha+\tan ^{2} \beta\right)^{-1 / 2}
\end{gathered}
$$

Since $\alpha$ and $\beta$ are fixed, $a_{1}, a_{2}$, and $a_{3}$ are also fixed. The $n_{1}, n_{2}$, and $n_{3}$ are the direction cosines of the normal vector $\bar{n}$ of the boundary surface, which is directed into the flow domain. Thus $A$ is a constant in Eq. 6. From the definition of the Riemann invariant $R^{-}$, the velocity magnitude is expressed as 


$$
V=\frac{1}{A}\left(R^{-}+\frac{2 c}{\gamma-1}\right)
$$

The Riemann invariant is evaluated from conditions from within the flow domain, and so, is the numerical condition required at the subsonic inflow boundary. Thus, $V$ is also only a function of the static temperature. With the static temperature determined from the iterations on Eq. 4, the Mach number, static pressure, static density, and velocity magnitude can easily be determined. The local flow angles determine the magnitudes of the velocity components.

For WIND, the local total conditions and flow angles can be specified as uniform values over the boundary or point specific as specified in the input data file or read from the boundary grid of the solution file.

\section{Bleed Boundary Conditions}

A bleed boundary condition is a subsonic outflow boundary, which requires one physical condition to be specified and four numerical conditions to be evaluated. It is a subsonic outflow even for supersonic core flows because the component of the flow normal to the surface is subsonic.

The basic premise of the bleed boundary conditions is the establishment of the bleed velocity defined as

$$
\vec{u}_{\text {bleed }}=-u_{\text {bleed }} \bar{n}
$$

where $\widehat{n}$ is the boundary normal surface vector, which is directed into the flow domain. For all of the bleed boundary conditions, $u_{\text {bleed }}$ is defined as

$$
u_{\text {bleed }}=\frac{\bar{m}_{\text {bleed }}}{\rho A_{\text {region }}}
$$

where $\rho$ is the density. The $A_{\text {region }}$ is the area over which the boundary condition is applied. The manner in which $\dot{m}_{\text {bieed }}$ is specified constitutes the physical boundary condition.

\section{Mass Flow Bleed Model}

The mass flow bleed model directly specifies $\dot{m}_{\text {bleed }}$. Options allow the specification of either the actual or corrected mass flow. One disadvantage of this boundary condition is that the bleed mass flow is fixed and can not adjust to local conditions, such as pressure changes due to shock motion.

\section{Porous Bleed Model}

The porous bleed model uses local flow conditions and some empirical data on the bleed system to allow the local bleed mass flow to vary. This boundary condition defines the bleed mass flow as

$$
\dot{m}_{\text {bleed }}=C_{d} \dot{m}_{\text {ideal }}
$$

where $C_{d}$ is a specified discharge coefficient whose value may be obtained from empirical data for the bleed system. The $\dot{m}_{\text {ideal }}$ is the ideal mass flow for an isentropic flow of air through a circular bleed hole

$$
\dot{m}_{\text {ideal }}=p_{t} A_{\text {bleed }} M\left(\frac{\gamma}{R T_{t}}\right)^{1 / 2} \phi^{-[(\gamma+1) / 2(\gamma-1)]}
$$

where

$$
\phi=1+\frac{\gamma-1}{2} M^{2}
$$

One can see that the ideal mass flow is a function of the local Mach number $M$ and the total conditions $p_{t}$ and $T_{t}$.

A bleed region is assumed to consist of some pattern slots or bleed holes. The $A_{\text {bleed }}$ is the cumulative area of the bleed holes or slots. It is related to area of the bleed region $A_{\text {region }}$ by the porosity $\Phi$ as

$$
A_{\text {bleed }}=\Phi A_{\text {region }}
$$

The condition holds that $0 \leq \Phi \leq 1$. For a slot covering the entire bleed region, $\Phi=1$.

One disadvantage of this boundary condition is that the value of the discharge coefficient $C_{d}$ is fixed during the simulation. It is known to usually be a function of the local flow conditions.

\section{Boeing Bleed Model}

The Boeing bleed model ${ }^{6}$ defines the bleed mass flow as

$$
\dot{m}_{\text {bieed }}=Q_{\text {sonic }} \dot{m}_{\max }
$$

where $Q_{\text {sonic }}$ is the sonic mass flow coefficient, which was empirically determined to be a function of the form

$$
Q_{\text {sonic }}=f\left(\alpha_{\text {bleed }}, M_{\text {local }}, \frac{p_{\text {plenum }}}{p_{t}}\right) .
$$


The $\alpha_{\text {bleed }}$ is the angle of the bleed hole with respect to the inlet surface. The $M_{\text {local }}$ and $p_{t}$ are the Mach number and total pressure at the edge of the boundary layer above the bleed hole. The $p_{\text {plenum }}$ is the static pressure of the bleed plenum, which is usually held constant in a bleed system. The empirical data for $Q_{\text {sonic }}$ was obtained from the implementation of this boundary condition in the NPARC code as described in Reference 6. Figure 1 shows the variation of $Q_{\text {sonic }}$. A bilinear interpolation is used to interpolate between the discrete empirical data. The effect of the $Q_{\text {sonic }}$ variation is that the bleed can turn off as the pressure ratios move towards unity. This prevents the bleed holes from blowing flow into the inlet.

The $\dot{m}_{\max }$ is the maximum mass flow determined by sonic conditions in the throat of the bleed hole, which is a "choked" bleed hole. The $\bar{m}_{\max }$ is evaluated from Eq. 13 with the Mach number $M=1$.

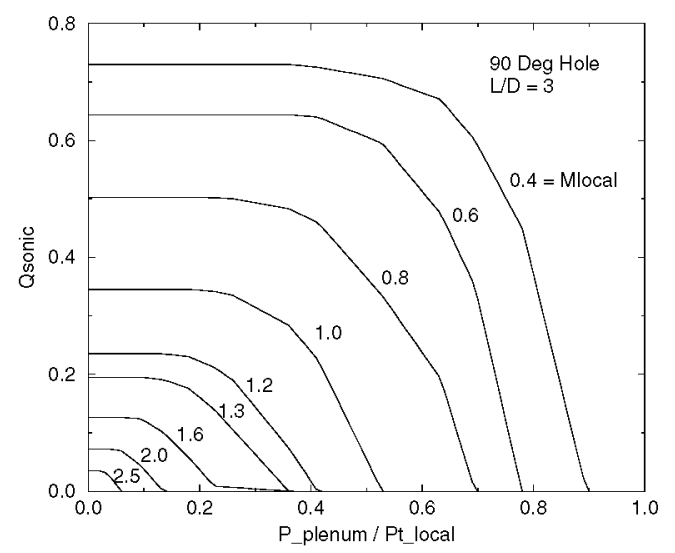

Figure 1. Variation of $Q_{\text {sonic }}$ with Mach number and pressure ratio for a $\alpha=90$-degree hole with a length / diameter ratio $\mathrm{L} / \mathrm{D}=3$.

\section{Subsonic Outflow Boundary Conditions}

The subsonic outflow boundary requires one physical condition to be specified and four numerical conditions to be evaluated. The numerical conditions are usually extrapolations of four of the conservative variables, usually density and the momentum components. In WIND, the physical boundary condition is the evaluation of the static pressure at the boundary in some reasonable manner. This is done using a variety of models, which behave differently for steady and unsteady flows. The models affect steady flow simulations in their convergence to the steady state and the characteristics of the flow near the outflow boundary. The models affect the unsteady flow simulations in their acoustic responses to flow disturbances interacting with the boundary.

\section{Specified Pressure Model}

The specified pressure model directly specifies the static pressure. It can be specified as steady or time varying and uniform or spatially varying.

\section{Mass Flow Model}

The mass flow model matches a specified mass flow by adjusting the static pressure through a relaxation with respect to the time-marching iterations

$$
p^{n+1}=p^{n}\left[1+\theta\left(\dot{m}_{B}-\dot{m}^{n}\right) / \dot{m}_{B}\right]
$$

where $\theta$ is the relaxation factor and $\dot{m}_{B}$ is the desired mass flow on the boundary. The mass flow may be the actual or corrected mass flow.

\section{Mach Number Model}

The Mach number model imposes a Mach number $M_{c f}$ at the boundary. ${ }^{7}$ The specified Mach number can be imposed locally or as an average on the boundary, which may indicate the expected Mach number at the compressor face. From the definition of total pressure, the static pressure at the boundary is

$$
p=p_{t}\left(1+\frac{\gamma-1}{2} M_{c f}^{2}\right)^{-\gamma /(\gamma-1)}
$$

where $p_{t}$ can be the local total pressure or an average over the outflow boundary.

\section{Nozzle Model}

The nozzle model uses the nozzle section of the verification inlet to vary the mass flow through the inlet. A choked nozzle operates at a constant mass flow and the boundary condition at its supersonic exit is a simple extrapolation of the conservative quantities. This approach has been used extensively in supersonic inlet applications for studies to determine the "mass flow cane" curve for the performance of inlets described by the variation of the total pressure recovery for a range of inlet mass flows. The mass flow is adjusted by varying the nozzle height $r$.

\section{Paynter Compressor Face Model}

The Paynter compressor face model is applied for unsteady flow applications and models the interactions 
of acoustic and convective disturbances with axial compressors. $^{8-10}$ The premise of the Paynter compressor face model is that the static pressure is a linear sum of acoustic and convective responses

$$
p^{n+1}=p^{n}+\delta p_{\text {acoustic }}+\delta p_{\text {convective }}
$$

The acoustic component has the general form of

$$
\delta p_{\text {acoustic }}=\sigma(1+\beta) \Delta p_{\text {disturbance }}
$$

where $\sigma$ is the CFL number and $\Delta p_{\text {disturbance }}$ is the incident pressure disturbance at the compressor face. The $\beta$ is the acoustic reflection coefficient defined as

$$
\beta=\Delta p_{\text {response }} / \Delta p_{\text {disturbance }} .
$$

The details of the model are presented in references 8-10. It should be noted that there are expressions for the other flow properties at the boundary that are used along with equation 20 . The model has built into it a non-reflecting option with $\beta=0$. Reference 10 discussed the implementation of this model into the NPARC CFD code. The current paper updates the presentation to show the implementation of this model into WIND.

\section{Verification Inlet}

A "parameterized" supersonic inlet has been devised for the current verification studies to allow the study of various geometric configurations. Figure 2 shows the various parameters defining the axial and transverse dimensions of the inlet. It is possible to generate planar flow domains for two-dimensional or axisymmetric simulations or three-dimensional flow domains with rectangular or axisymmetric cross-sections.

The inlet consists of five sections: foreduct, ramp, diffuser, isolator, and nozzle. The foreduct, isolator, and nozzle can each be removed independently from the flow domain. The lower surface of all of the sections is straight with a dimension of $\mathbf{g}$ units. For a three-dimensional, axisymmetric domain, lower surface becomes a constant-radius surface of $\mathbf{g}$ units. The foreduct has a length of a units and height of $\mathbf{h}$. The foreduct is a straight section ahead of the ramp section and is used to generate a boundary layer or place the inflow boundary condition a certain distance forward of the ramp. The ramp section has a length of $(\mathbf{b}+\mathbf{c})$ units. The length $\mathbf{c}$ denotes the bleed region on the lower surface. The ramp can deflect down to generate oblique shocks in supersonic flow or deflect up to generate an expansion wave in supersonic flow or a conical expansion surface in subsonic flow. The diffuser section transitions the duct to the exit height of $\mathbf{o}$ units over a length of $\mathbf{d}$ units. The upper surface of the diffuser is defined as a cubic spline with zerotangent slopes at the start and end. A compressor face would nominally be placed at the end of the diffuser. The isolator is a constant-height section meant to place the outflow boundary condition farther downstream of the diffuser exit. Such an approach attempts to minimize the local effects of the boundary condition on the flow at the compressor face. The isolator has a length of e units. The nozzle is used as one model for a subsonic outflow boundary. The height of the nozzle throat is $\mathbf{r}$ units.

A Fortran program was written to automatically generate the geometry, grid, and boundary condition setup files for the simulations. The grid was generated based on specified grid axial and wall spacings and grid quality controls. The grids are structured H-grids consisting of a single zone. Axial grid planes are at constant-x coordinates. Inputs allow global control of the grid spacings to allow for easy scaling of the grid sizes for use with grid convergence studies.

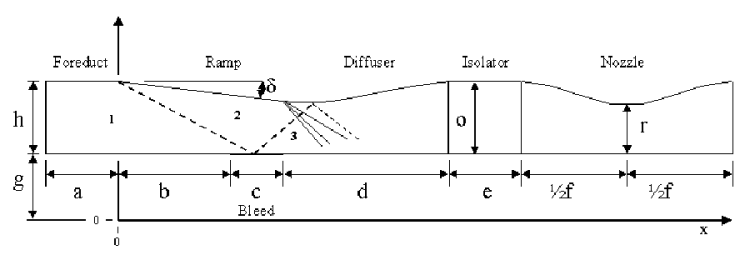

Figure 2. Verification inlet with parameters.

\section{Computational Studies}

Computational studies were performed using the verification inlet for various geometric configurations and flow conditions. The objective of the studies was to examine the behavior of the boundary conditions under relatively simple circumstances with the emphasis on verification. Each study examines aspects of one or more of the boundary conditions. The studies are discussed in the following sections.

\section{Uniform Mach 1.3 Flow in a Straight Duct}

This study examined the uniform flow of Mach 1.3 in a straight duct with a boundary layer formed on the bottom surface of the inlet. Figure 3 shows the flow domain along with the Mach number contours and lists the values of the geometric parameters in units of feet. The flow domain is a straight, two-dimensional duct of width 1.0 feet and does not contain an isolator or nozzle. Mach 1.3 is a common Mach number ahead of the normal shock dividing the supersonic and subsonic flows in a supersonic inlet. It is considered the 
minimum Mach number to ensure a stable transition to subsonic flow without a too great of loss of total pressure across the shock. A bleed region extends from $x=2.0$ to $x=3.0$ feet and extracts some of the flow from the boundary layer. The objective is to examine and compare the behavior of the bleed models in uniform flow. Since the flow is supersonic, the inflow boundary condition is fixed and the outflow boundary condition is an extrapolation of the conservative variables. Thus, the bleed boundary condition is essentially isolated from the inflow and outflow boundary conditions.

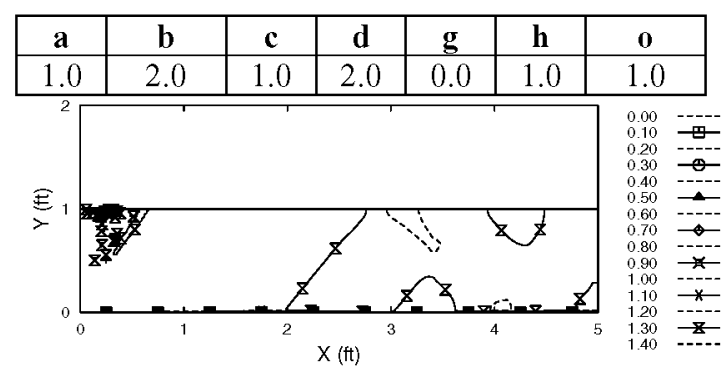

Figure 3. Flow domain, Mach number contours, and geometric parameters for the uniform flow of Mach 1.3 in a straight duct.

A grid convergence study was performed to examine the effects of grid spacing on the bleed mass flow. Simulations were performed on five grids for which the grid refinement ratios were $r=1.5$. The simulations used the Boeing bleed and Spalart-Allmaras turbulence models. The resulting order of convergence was $p=$ 1.419. Figure 4 shows the variation of the bleed mass flow with respect to the normalized grid spacing. The plot shows a predicted zero-grid value of bleed flow of $2.169 \%$. The $G C I$ values were $0.66 \%, 1.17 \%, 2.04 \%$, and $3.96 \%$ over the pairs of successively coarser grids. This suggests an error band of $0.014 \%$ for the vlaue of bleed mass flow. The asymptotic nature of the simulated bleed flows is observed in Fig. 5. The ratios of the $G C I$ values indicated that the simulations on four finest grids were within the asymptotic range.

Simulations were performed on the medium grid of the grid convergence study for the various bleed models and turbulence models. Figure 5 shows the character of the bleed flow in the form of the streamwise distribution of the mass flow along the duct across the bleed region. Shown are the results of simulations using the Spalart-Allmaras and SST turbulence models. The simulation using the Boeing bleed model with the Spalart-Allmaras model was performed first and resulted in a total mass flow bleed of $2.215 \%$ of the captured mass flow. The simulation using the porous bleed model was then performed using a discharge coefficient that was adjusted until the mass flow bleed was also $2.215 \%$. The simulation using the mass flow bleed model directly specified the bleed mass flow to be $2.215 \%$. Verification of the mass flow bleed model was a simple check that the computed bleed was the same as the specified bleed, which it was. Figure 5 shows that for uniform flow, all three models performed the same, which one would expect. The variation is linear, which indicates that each boundary grid point bleeds the amount of flow proportional to its cell-face area. The Boeing and porous models perhaps have a slight curvature indicating a slightly higher bleed rate at the downstream portion of the bleed region.

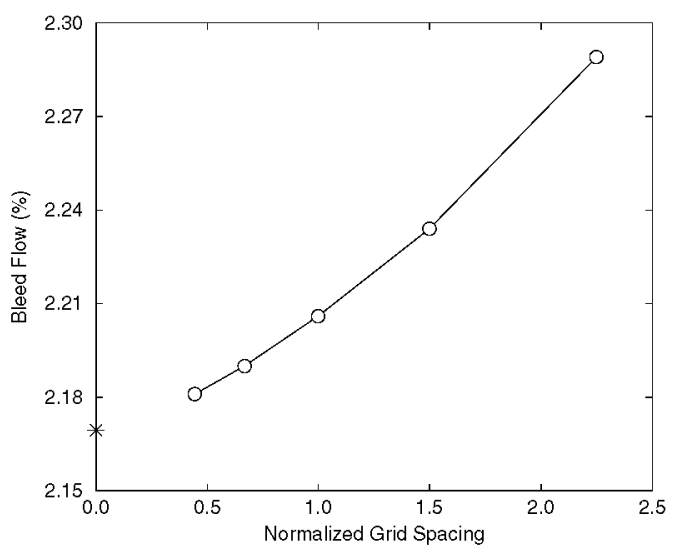

Figure 4. Variation of the bleed mass flow over a series of finer grids.

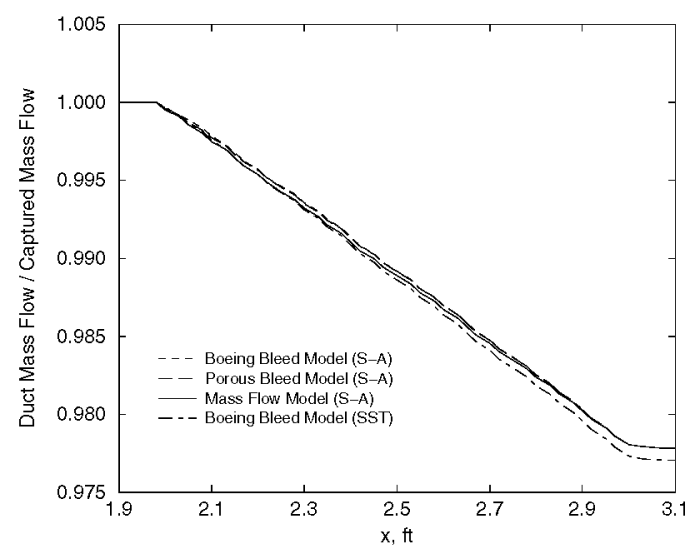

Figure 5. Streamwise distribution of the mass flow along the duct over the bleed region in a uniform flow of Mach 1.3 with a $2.215 \%$ bleed.

Simulations using the Baldwin-Lomax and Chien k- $\varepsilon$ turbulence models resulted in the same mass flow as the simulation using the Spalart-Allmaras. The simulation using the SST indicated a total bleed mass flow of 
$2.298 \%$, which is $3.75 \%$ higher than the simulations using the Spalart-Allmaras. The reasons for this difference are not yet clear.

\section{Normal Shock Interacting with a Bleed Region}

This study examined the flow of Mach 1.3 in a straight duct in which a normal shock interacts with the bleed region. A normal shock is often used in supersonic inlets to decelerate the flow from supersonic to subsonic speeds. Bleed is used to stabilize the movement of the normal shock and minimize adverse effects of shock/boundary interaction.

The geometry and flow conditions of this study were the same as the previous study; however, for this study a nozzle section was used. Choking the flow in the nozzle generates a normal shock. The forward motion of the normal shock is due to the imbalance of the captured mass flow ahead of the shock and the lesser mass flow behind the shock going through the nozzle throat. This condition is essentially a hammershock. Since the flow is choked at the nozzle throat, the mass flow can be adjusted by varying the throat height $r$ of the nozzle. Figure 6 shows the flow domain for this study. The Mach number contours of Fig. 6 show the normal shock in the bleed region, which is from $x=2.0$ to $x=3.0$ feet.

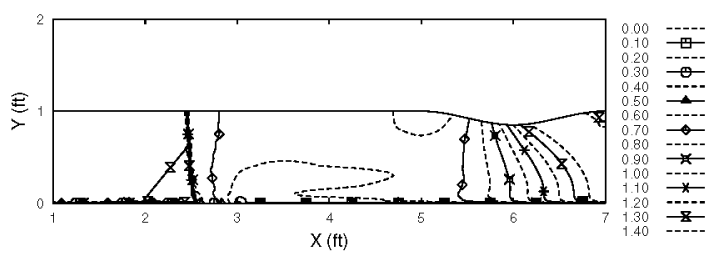

Figure 6. Flow domain and Mach number contours for a normal shock positioned over a bleed region.

This study shows the capability of a bleed boundary condition that can adjust its bleed mass flow according to local flow conditions, such as the Boeing bleed model. Once the shock moves into the bleed region, the forward portion of the bleed region is under a supersonic flow condition while the rearward portion of the bleed is under a subsonic flow, which is at a higher pressure. In a bleed stability system, the bleed plenum pressure is usually held constant. A higher duct pressure, as found behind the shock, would cause an increase in the bleed flow. This has a stabilizing effect on the shock since now the excess captured mass flow can be directed out through the bleed region. If enough mass flow is removed through the bleed, the normal shock position can be stabilized.
Simulations were performed using the Boeing bleed model in which the nozzle throat heights were varied to produce a range of nozzle mass flows. The simulations used the Spalart-Allmaras turbulence model. The normal shock moved into the bleed region and the Boeing bleed model was able to stabilize the normal shock and a steady-state flow solution was obtained. Figure 7 plots the variation of the bleed mass flow with nozzle throat mass flow. As can be seen, as the nozzle mass flow decreased (smaller nozzle throat area), the bleed mass flow increased. Two observations are that the variation is linear and the sum of the bleed mass flow and the nozzle throat mass flow percentages is $100 \%$. The variation will intersect the $0 \%$ bleed mass flow at a $100 \%$ nozzle throat mass flow. Figure 8 shows the streamwise distribution of the mass flow over the bleed region for the simulation at which the normal shock is positioned at approximately $x=2.5$ feet. One can see the "kink" in the curve at the shock location and an increase in the mass flow behind the shock. Shown are the distributions for the simulations using the Spalart-Allmaras and SST turbulence models, which behave in a similar manner.

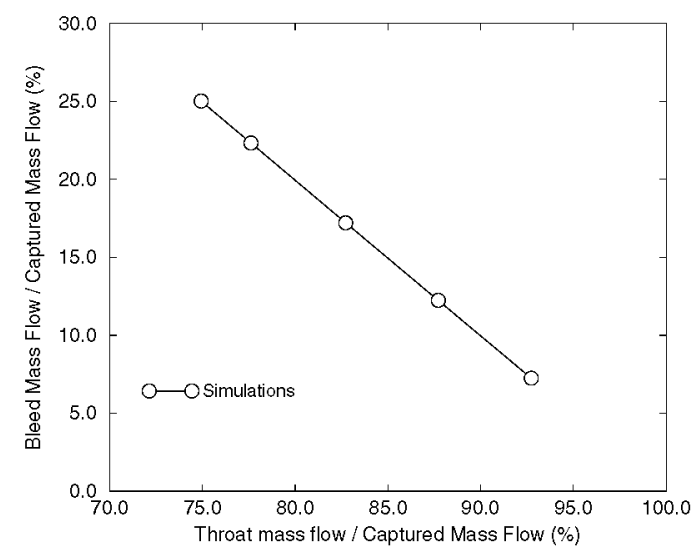

Figure 7. Streamwise variation of the bleed mass flows with various nozzle throat mass flows for a normal shock positioned over the bleed region modeled with the Boeing bleed model.

The mass flow and porous bleed models were unable to stabilize the shock. The mass flow bleed model holds the total mass flow fixed, and so, was unable to increase the mass flow through the bleed region. The porosity bleed model had no mechanism to adjust the discharge coefficient. Both bleed models resulted in the unstart of the inlet, which was characterized by the normal shock moving ahead of the bleed region. 


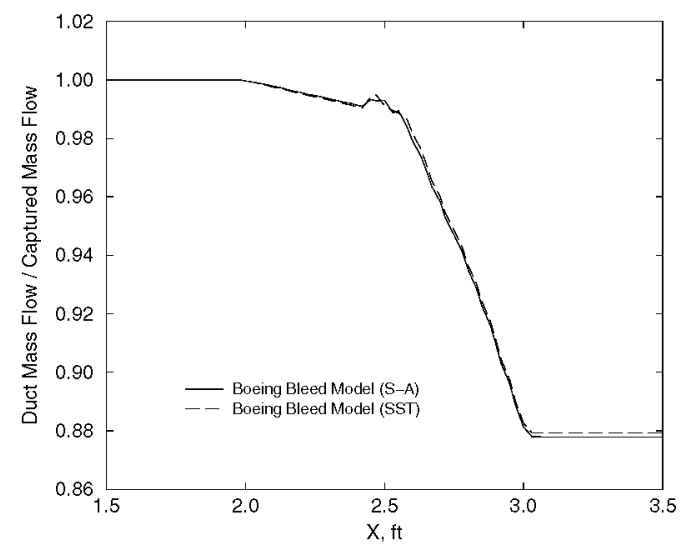

Figure 8. Streamwise distribution of the bleed mass flow along the duct over the bleed region with a normal shock positioned over the bleed region.

\section{Oblique Shock / Bleed Interaction}

This study examined the flow of Mach 2.35 with an oblique shock generated by a 10 -degree ramp that reflects off and interacts with a bleed region on the lower wall of the inlet. This flow condition is illustrated in Fig. 2. Figure 9 shows part of the flow domain in the area of the ramp and bleed region along with the Mach number contours. Figure 9 lists the values of the geometric parameters for this study in units of feet. The foreduct length $\boldsymbol{a}$ was chosen long enough to generate a boundary layer in the bleed region such that a separation bubble developed when no bleed was used. The resulting boundary layer thickness ahead of the bleed region was approximately 0.1 feet. The ramp was positioned such that the oblique shock impinged at the center of the bleed region, $x=1.5$ feet. The domain does not include an isolator or nozzle. The domain and grids were two-dimensional with a specified width of 1.0 feet.

\begin{tabular}{|c|c|c|c|c|c|c|}
\hline$a$ & $b$ & $c$ & $d$ & $g$ & $h$ & $o$ \\
\hline 7.0 & 1.252 & 0.5 & 2.0 & 0.5 & 1.0 & 0.821 \\
\hline
\end{tabular}

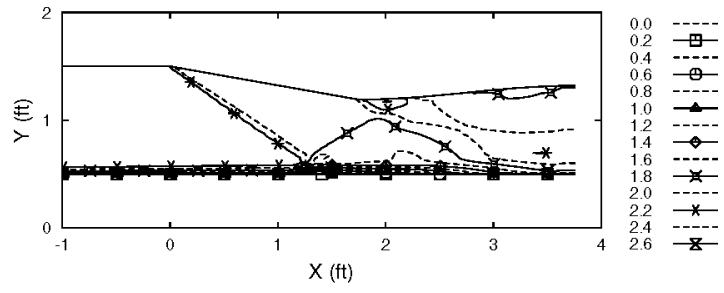

Figure 9. Flow domain, Mach number contours, and geometric parameters for an oblique shock interacting with a bleed region in Mach 2.35 flow.
The objective of the study was to create varying conditions over the bleed region, but not those as severe as for the normal shock study. Such oblique shock reflections exist in supersonic inlets. The bleed attempts to minimize the separation due to shock/boundary layer interactions.

A simulation was performed assuming inviscid flow so as to compare with inviscid, steady flow theory. Figure 10 shows the comparison. Shown is the step decrease in Mach number across the oblique shock on the upper surface and the reflected shock on the lower surface. The comparison is good.

A grid convergence study was conducted to examine the variation of mass flow bleed with respect to grid refinement. Three grids were used with a grid refinement ratio of $r=2.0$. The Boeing bleed and the Spalart-Allmaras turbulence models were used. A significant amount of variation was observed such that a negative value of the order-of-convergence $p$ was calculated. When a value of $p=1.5$ was assumed, the $G C I$ values ranged from $4.2 \%$ on the coarse grid to $11.6 \%$ on the fine grid.

Simulations were performed using medium grid with the Spalart-Allmaras turbulence model and varying the three bleed models. The Boeing and porous bleed models each used the same bleed plenum pressure. The discharge coefficient of the porous bleed model was adjusted until the total bleed flow was the same as that of the Boeing bleed model, which was $1.838 \%$. This amount of bleed flow was then specified for mass flow bleed model. Figure 11 shows the streamwise distribution of the mass flow across the bleed region. The mass flow bleed model resulted in a linear distribution. The Boeing bleed model essentially does not bleed any flow upstream of the shock. The porous bleed model actually injects some flow prior to the shock, but then bleeds the flow downstream of the shock to get to the same level of total bleed.

Simulations using other turbulence models showed some variation in the bleed mass flow. The simulation using the SST turbulence model resulted in the lowest bleed mass flow of $1.662 \%$.

Three simulations were also performed using the wall function with the Spalart-Allmaras turbulence model. The medium grid was used with sublayer grid points removed at $y+$ values of 28,51 , and 94 . The bleed mass flow remained fairly constant at $1.84 \%$ for all three simulations.

A simulation was performed with a threedimensional domain and grid with a rectangular crosssection and a width of 1.0 feet. It was verified that the bleed mass flow was identical to that of the twodimensional domain and grid. 


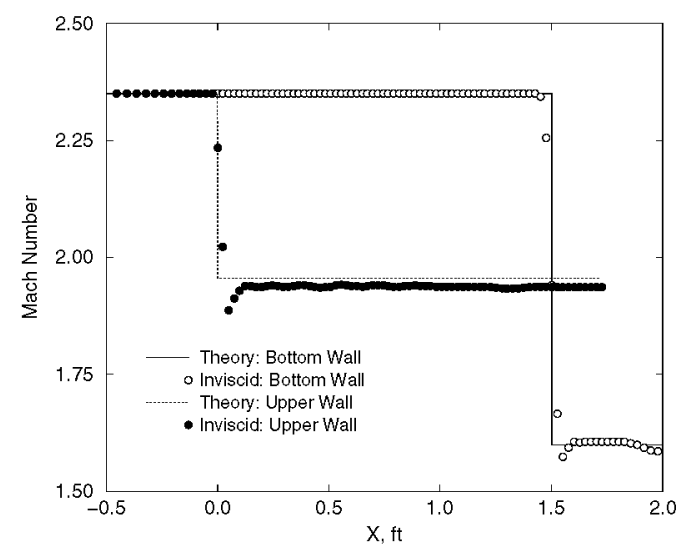

Figure 10. Comparison of surface Mach numbers with theory for inviscid, Mach 2.35 flow with an oblique shock and no bleed.

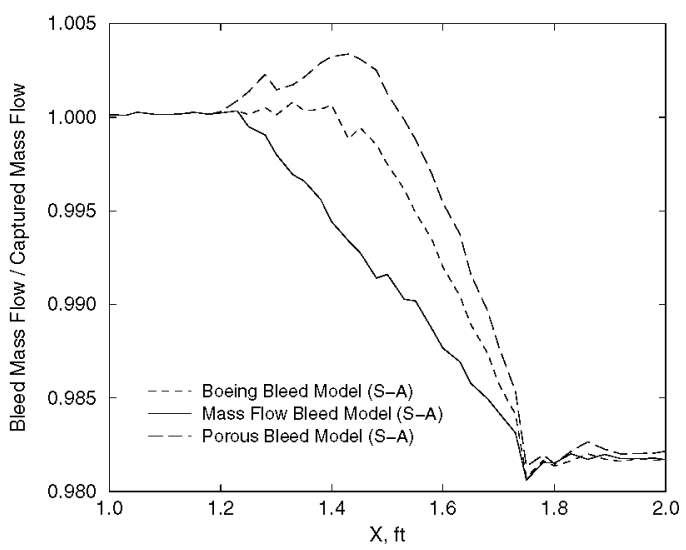

Figure 11. Streamwise distribution of mass flow in the duct across the bleed region with an oblique shock in Mach 2.35 flow interacting with the bleed.

\section{$\underline{\text { Subsonic Diffuser Duct }}$}

This study examined the steady, subsonic flow in a diffuser duct. The objective was to verify the operation of the subsonic inflow and subsonic outflow boundary conditions. Figure 12 shows the flow domain, Mach number contours, and the values of the geometric parameters in units of feet. The flow domain is axisymmetric. The subsonic inflow boundary condition was applied at the inflow to hold fixed the inflow total pressure, total temperature, and flow angles, which are directed axially. The subsonic outflow boundary conditions are applied at the outflow. The area distribution and boundary conditions result in a Mach 0.35 outflow and a Mach 0.786 inflow for the inviscid flow simulation. The Mach 0.786 inflow is the Mach number behind a normal shock with a Mach 1.3 inflow and is a typical design Mach number at the entrance to the subsonic diffuser of a supersonic inlet. The Mach 0.35 outflow is a typical design Mach number at the entrance to the compressor face of the gas turbine engine.

\begin{tabular}{|c|c|c|c|c|c|c|}
\hline$a$ & $b$ & $c$ & $d$ & $g$ & $h$ & $o$ \\
\hline 1.0 & 0.5 & 0.5 & 5.0 & 0.0 & 1.0 & 1.305 \\
\hline
\end{tabular}

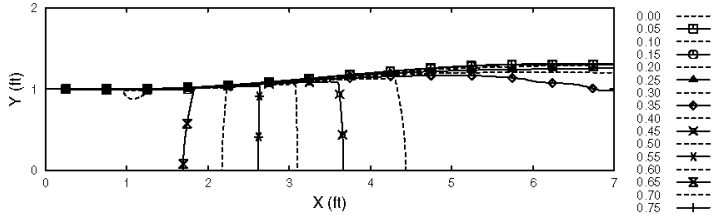

Figure 12. Flow domain, Mach number contours, and geometric parameters for the axisymmetric flow in a subsonic diffuser duct.

Simulations were performed assuming a steady, inviscid flow. Verification of the subsonic inflow boundary condition involved a straightforward check on whether the total pressure and total temperature at the inflow boundary matched that, which was specified in the input file. A check indicated that there was a match to seven significant digits. Verification of the solution involved a comparison with the analytic solution for the axial distribution of the Mach number through the duct as determined through quasi-one-dimensional theory. ${ }^{\mathbf{1}}$ Figure 13 shows a good comparison. Simulations were performed with both planar and three-dimensional axisymmetric domains and comparisons verified the equality of both domains. Verification of the subsonic outflow boundary conditions involved checking whether the outflow Mach number was 0.35, the outflow static pressure matched the value specified, and the outflow mass flow matched the specified value, which all did within $0.1 \%$. Simulations were performed using the mass flow, Mach number, and nozzle boundary conditions. The results agreed with the use of the constant-pressure boundary condition.

Simulations were performed assuming turbulent flow. The Spalart-Allmaras turbulence model was used with constant-pressure, Mach number, and mass flow boundary conditions. An axisymmetric simulation matched the mass flow and exit Mach number of a three-dimensional, axisymmetric simulation. The length of the isolator affects the conditions at the compressor face with respect to the type of outflow boundary condition. 


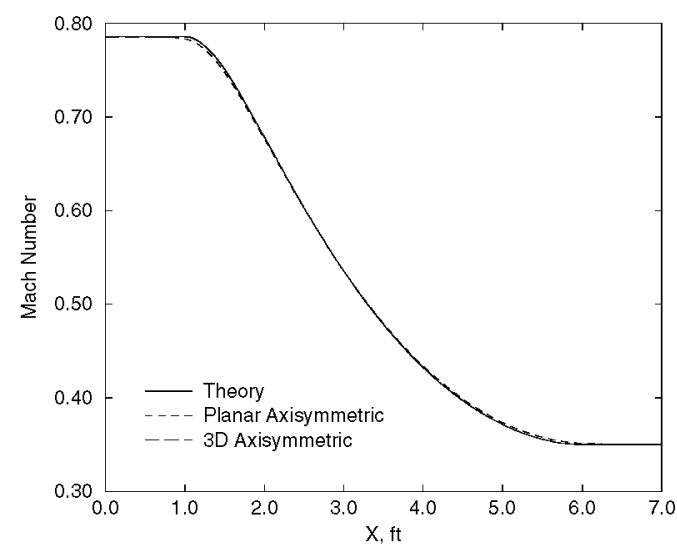

Figure 13. Distribution of Mach number along the subsonic diffuser duct in comparison to theory.

\section{$\underline{\text { Step Acoustic Disturbance in an Annular Duct }}$}

This study examined the unsteady, inviscid flow in a straight, annular duct with a step acoustic disturbance propagating down the duct and interacting with the subsonic outflow boundary condition. The objective was to examine the unsteady acoustic response from the subsonic outflow boundary conditions. The simulation of such responses are of importance in supersonic inlet design with respect to the stability of the inlet flow. Figure 14 shows the flow domain and Mach number contours for the study at the start of the simulation. The step acoustic disturbance had a magnitude of $+10 \%$ of the nominal static pressure and was initially located from $x=0$ to 5 inches. The values of the geometric parameters in units of inches associated with this study are listed in Fig. 14. The flow domain is an axisymmetric, annular duct. The subsonic inflow boundary condition was applied at the inflow to hold fixed the inflow total pressure, total temperature, and flow angles, which are directed axially. The subsonic outflow boundary conditions were applied at the outflow.

\begin{tabular}{|c|c|c|c|c|c|c|}
\hline$a$ & $b$ & $c$ & $d$ & $g$ & $h$ & $o$ \\
\hline 1.0 & 10.0 & 5.0 & 5.0 & 0.5 & 1.0 & 1.0 \\
\hline
\end{tabular}

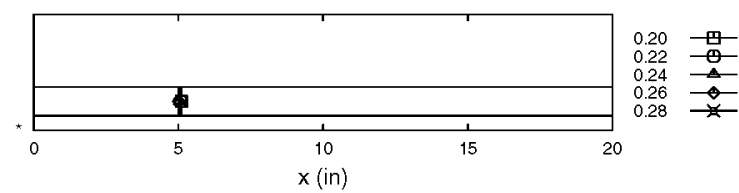

Figure 14. Flow domain, Mach number contours, and geometric parameters for flow with a $10 \%$ acoustic disturbance in an annular duct.
Verification of the subsonic inflow boundary condition involved a straightforward check on whether the total pressure and total temperature at the inflow boundary matched that, which was specified in the input file. A check indicated that there was a match to seven significant digits.

Verification of the subsonic outflow boundary condition models involved examining the propagation of the acoustic disturbance and the reflection of the acoustic response of the models to the step acoustic disturbance. Figure 15 shows the time-varying static pressure as observed at a sensor position located at $\mathrm{x}=$ 15.0 inches. The propagation speed of the downstream-traveling acoustic disturbance is $u+c$ where $u$ is the axial flow speed and $c$ is the acoustic speed. Thus, the disturbance reaches the sensor location at $0.621 \mathrm{msec}$. The propagation speed of the upstream-traveling acoustic response is $u-c$. Thus, the response reaches the sensor location at $1.434 \mathrm{msec}$. Figure 15 shows that the simulations do propagate the disturbance and responses at the correct speeds. The behavior of the subsonic outflow boundary conditions with respect to the acoustic responses is consistent with the respective boundary condition. The pressure model imposes a constant pressure at the outflow, and so, the response is equal to the specified pressure. The Mach number model imposes the constant Mach number, and so, the response is of the same sign and magnitude as the disturbance. The Paynter compressor face model gives a response of the same sign, but of less magnitude as the disturbance. The non-reflecting model (Paynter Model with $\beta=0$ ) essentially gives no response, although a slight reflection is noticed and the reasons for this are still under study.

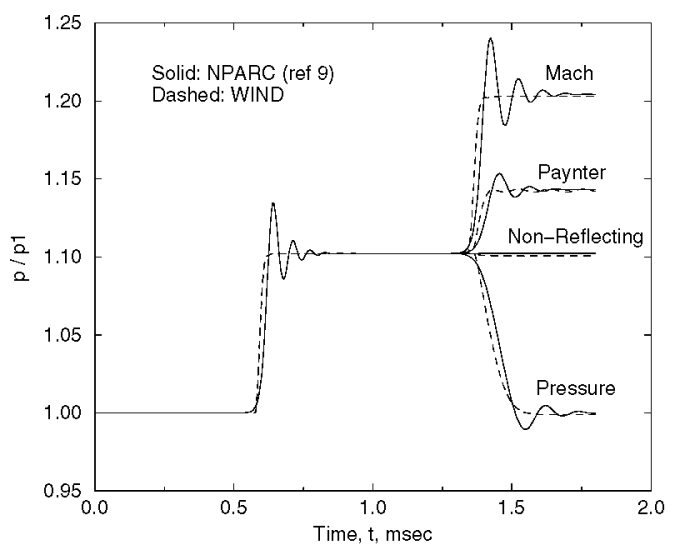

Figure 15. Response from the outflow boundary condition to an acoustic disturbance in a straight annular duct. 
Figure 15 also shows the time varying pressures as obtained from simulations using the NPARC CFD code, which were originally presented in reference 10 . The two codes agree well, but WIND removes oscillations at the pressure discontinuities.

\section{Summary and Conclusions}

Boundary conditions for subsonic inflow, bleed, and subsonic outflow used for computational analysis of the flow through supersonic inlets have been discussed and assessed with respect to verification. The relatively simple configurations and flow simulations were demonstrated to be powerful in providing important information on the behavior of the boundary conditions. However, verification is a never-ending process that tends to create numerous issues for further examination.

\section{$\underline{\text { References }}$}

1. Bush, R.H., G.D. Power, and C.E. Towne, "WIND: The Production Flow Solver of the NPARC Alliance," AIAA Paper 0935, January 1998.

2. WIND Code web site,

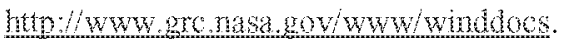

3. AIAA, "Guide for the Verification and Validation of Computational Fluid Dynamics Simulations," AIAA G-077-1998, 1998.
4. Roache, P.J., Verification and Validation in Computational Science and Engineering, Hermosa Publishers, Albuquerque, New Mexico, 1998.

5. NPARC Verification and Validation web site,

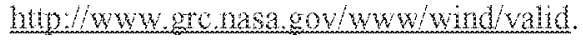

6. Mayer, D.W. and G.C. Paynter, "Boundary Conditions for Unsteady Supersonic Inlet Analyses," AIAA Journal, Vol. 32, No. 6, June 1994, p. 1200-1206.

7. Chung, J.K. and Cole, G.L., "Comparison of Compressor Face Boundary Conditions for Unsteady CFD Simulations of Supersonic Inlets," AIAA Paper 95-2627.

8. Paynter, G.C., "Modeling the Response From a Cascade to an Upstream Convective Velocity Disturbance," AIAA Paper 98-3570.

9. Paynter, G.C., L.T. Clark, and G.L. Cole, "Modeling the Response from a Cascade to an Upstream Acoustic Disturbance," AIAA Journal, Vol. 38, No. 8, August 2000, p. 1322-1330.

10. Slater, J.W. and G.C. Paynter, "Implementation of a Compressor Face Boundary Condition Based on Small Disturbances," NASA/TM-2000-209945, March 2000. (Also ASME 2000-GT-0005).

11. Anderson, J.D., Modern Compressible Flow, McGraw-Hill, 1984. 


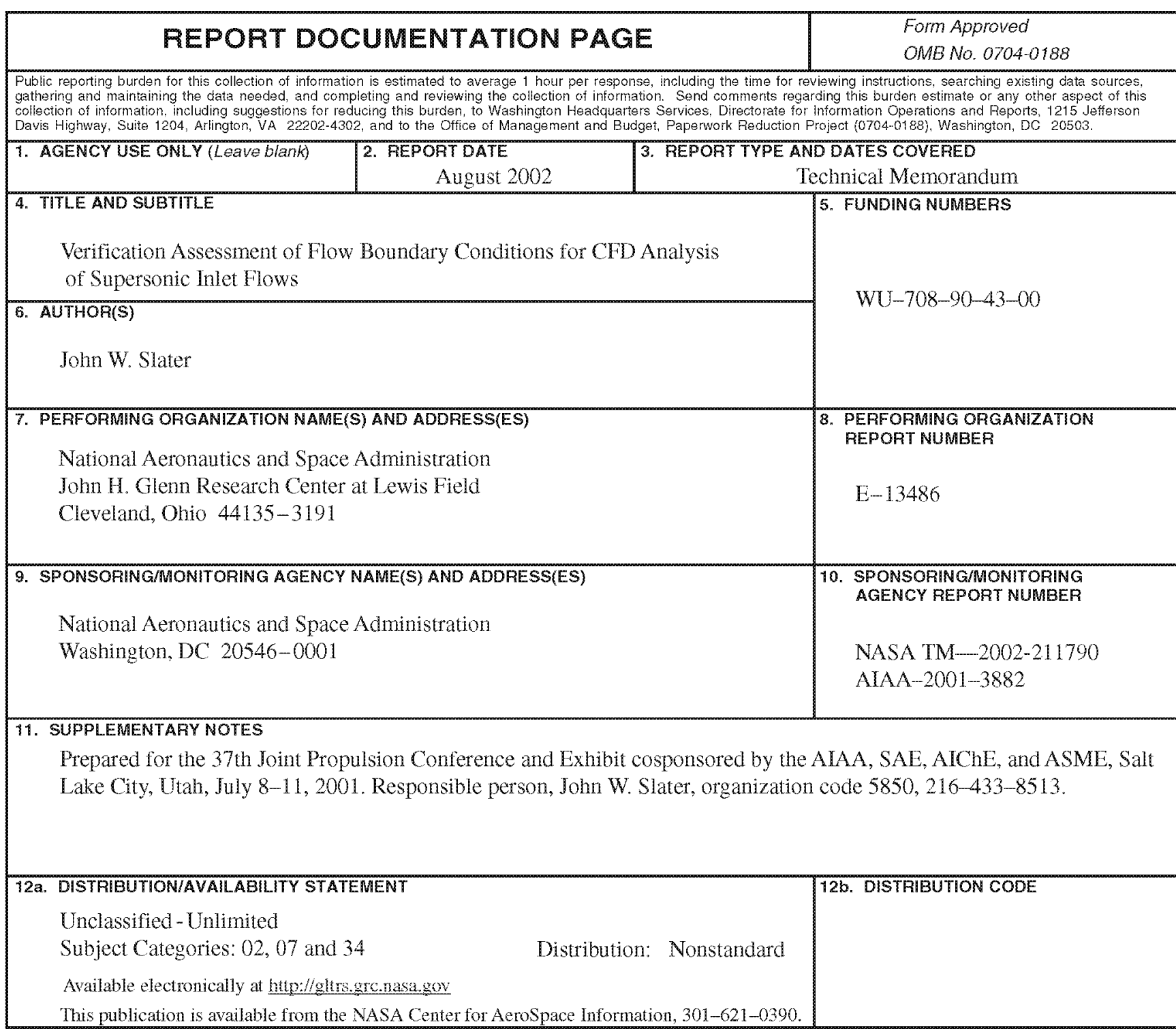

13. ABSTRACT (Maximum 200 words)

Boundary conditions for subsonic inflow, bleed, and subsonic outflow as implemented into the WIND CFD code are assessed with respect to verification for steady and unsteady flows associated with supersonic inlets. Verification procedures include grid convergence studies and comparisons to analytical data. The objective is to examine errors, limitations, capabilities, and behavior of the boundary conditions. Computational studies were performed on configurations derived from a "parameterized" supersonic inlet. These include steady supersonic flows with normal and oblique shocks, steady subsonic flow in a diffuser, and unsteady flow with the propagation and reflection of an acoustic disturbance.

\begin{tabular}{|c|c|c|c|}
\hline & 14. SUBJECT TERMS & & $\begin{array}{c}\text { 15. NUMBER OF PAGES } \\
18\end{array}$ \\
\hline \multicolumn{3}{|c|}{ Computational fluid dynamics; Boundary conditions } & 16. PRICE CODE \\
\hline $\begin{array}{l}\text { 77. SECURITY CLASSIFICATION } \\
\text { OF REPORT } \\
\text { Unclassified }\end{array}$ & $\begin{array}{l}\text { 18. SECURITY CLASSIFICATION } \\
\text { OF THIS PAGE } \\
\text { Unclassified }\end{array}$ & $\begin{array}{l}\text { 19. SECURITY CLASSIFICATION } \\
\text { OF ABSTRACT } \\
\text { Unclassified }\end{array}$ & 20. LIMITATION OF ABSTRACT \\
\hline NSN $7540-01-280-5500$ & & & $\begin{array}{l}\text { andard Form } 298 \text { (Rev. 2-89) } \\
\text { scribed by ANSI Std. Z39-18 } \\
\text {-102 }\end{array}$ \\
\hline
\end{tabular}

